\title{
A Short Analysis of Merge Order*
}

\author{
Zhiyi Zhang \\ School of Foreign Languages and Cultures, Nanjing Normal University, Nanjing, Jiangsu Province, China
}

\begin{abstract}
Merge is the basic syntactic operation for generative and transformative syntax. Ever since the initial stage of G-T, the bottom-up merge order or linearly leftward merge order is advocated. However, the present study aims at providing syntactic evidence against this bottom-up merge order and advocates a rightward merge order.
\end{abstract}

Index Terms - merge order, bottom-up merge order, syntactic evidence, rightward merge order

\section{INTRODUCTION}

There are many theoretical studies concerning different aspects of the syntactic operation of merge, which is defined as the basic syntactic operation for syntactic numeration. The most frequently debated topic concerning merge will be merge order issue, which will also be the focus of the present merge study. Experts have different ideas. The traditional and prevalent one would be bottom-up merge order or the leftward merge order. Besides Chomsky, almost all the other important figures in G-T syntactic study advocate a bottom-up merge order and typically Andrew Radford, in his up-to-date work Minimalist Syntax: Exploring the Structure of English, reemphasizes the importance of bottom-up or leftward merge order and derives sample sentences strictly according to the order(Andrew,2008). And a Chinese figure especially deserving our attention will be Dai Manchun. His theory of leftward merge in broad sense best revealed in his doctoral dissertation Leftward Merge in Broad Sense not only reconfirms the importance of leftward merge order but also intends to prove that all the adjuncts are inserted into the derivation from left instead of the traditionally acknowledged insertion from right, he first makes it clear that bottom-up merge order is a principle that all derivations must strictly follow (Dai, 2001).

\section{Syntactic Evidence Against Bottom-uP}

The G-T syntax, ever since its beginning stage till the recent theory has been concentrating on the logical and semantic relation between lexical items and the syntactic representations of this relation. In the early stage of the G-T theory, the logical-semantic relations are represented in deep structure and its syntactic representation can be observed in surface structure. In recent theories especially in phase theory, when derivational pattern replaces representational pattern, the logical semantic relation and its syntactic representation are realized simultaneously through the basic syntactic operation of merge or move. In this sense, merge order ought to match the logical semantic relation and the concerning syntactic representations. Among the syntactic representations of logical semantic relations, case, agreement, government and binding are the prime considerations for G-T studies. A careful examination of these syntactic elements testifies that if a bottom-up merge order is observed, these syntactic elements fail to establish the proper logical semantic relation. The present study will examine them separately.

\section{A. Evidence of Case}

Chomsky(1981), following an idea suggested by Jean-Roger Vergnaud, proposed that case should be regarded as the prerequisite for DP to be active in syntax. That is to say, a sentence containing any DP without case appropriate for its structural position is excluded as an ungrammatical one. This is so called Case Filter condition and Chomsky also defines some case assignment rules.

Case Filter

*NP if NP has phonetic content and has no case.

Case assignment rules

NP is nominative if governed by AGR

$\mathrm{NP}$ is objective if governed by $\mathrm{V}$ with the sub-categorization feature:

NP (i.e., transitive)(Andrew,2004,P.367)

In his earlier theory, Chomsky supposes that Agr category assigns nominative case to NP while a transitive $\mathrm{V}$ assigns objective case to NP. ( During this earlier stage, Chomsky once even suggested two Agr categories, AgrS and AgrO to assign nominative and objective case separately.) And in minimalism and phase theory because Agr category has been cancelled in syntactic operation and replaced by $\mathrm{T}, \mathrm{T}$ is in charge of assigning nominative case to NP as can be seen in Radford's derivation of the sentence Will Ruritania withdraw the troops from Utopia?(Andrew,2004).

\footnotetext{
* The present research is supported by the Humanity and Social Sciences Research Fund for the Youth from Ministry of Education of China (Grant No. 13YJC740144).
} 
The present study here posits that if a bottom-up merge order is followed, it will cause problems for case assignment. First the assignment of accusative case will be examined.

In accordance with the bottom-up merge order, a new syntactic element is merged from left to the existing syntactic element. At the bottom of the hierarchical structure, when transitive verb and NP merge, first there will be an NP and then transitive verb merges from left to the existing NP. This will lead to a problem in accusative case assigning. It is known that no matter in minimalism or in phase theory, a standard assumption for language derivation is that a language consists of two components: a lexicon and a computational system. The lexicon specifies the lexical items that enter into the computational system, with their idiosyncratic properties (Chomsky, 2001). The computational system uses these elements to generate derivations. The derivation of a particular linguistic expression then involves a choice of items from the lexicon and a computation that constructs the pair of interface representations.

When the bottom-up merge order is followed, the computational system will first choose NP from lexicon to make it an existing syntactic element for further syntactic operation. Once NP is chosen from the lexicon and enters derivation, it has been specified and its idiosyncratic properties should be identified. Among these idiosyncratic properties, case feature is an important one because in some languages like English, case feature will directly influence the phonetic content of NP. On the contrary, according to the case assignment rules, the accusative case should be assigned by transitive verb while at this time the transitive verb has not entered the derivation yet. Naturally this causes a dilemma. As a lexical term chosen from the lexicon, all its properties have been determined. But its case feature has not been assigned yet.

The only way to settle this dilemma is to change bottom-up merge order by adopting an opposite one. In that case, the computational system first chooses a transitive verb from the lexicon to form an existing syntactic element. This transitive verb can utilize its own competence of assigning accusative case to predetermine the case feature and the concerning phonetic content of NP. Once this has been pre-determined, the computational system can then choose NP from lexicon to merge transitive verb. Only in this order can the accusative assigning problem be successfully solved.

As far as the nominative case is concerned, the situation is more complicated. As has been mentioned in the above section, in the early stage of case theory, the nominative case of an NP is assigned by Agr and in the recent case theory, a broader category $\mathrm{T}$ is defined to indicate tense, match person, number feature and assign case feature. If so, the same problem will emerge. In a typical derivation of a sentence, to satisfy the UTAH(Andrew, ( if UTHA is accepted as a presupposition), NP used as the subject of the sentence is first located at the inner specifier position of $\mathrm{v}$ and then through move, NP finally occupies the outer specifier position of T.In another word, in the derivation, NP enters derivation before $\mathrm{T}$, which is in charge of assigning a nominative case to NP itself. This causes another dilemma which is once again caused by bottom-up merge order and therefore, in order to solve the problem, $\mathrm{T}$ enters derivation first and assigns nominative case to NP and predetermines the phonetic content of NP. Then NP is chosen by the computational system to enter derivation and merges $\mathrm{T}$ and NP can still be at the inner specifier position and satisfies UTAH. As a result, the problem seems to be smoothly solved by advocating an opposite merge order.

But the problem is not so simple concerning the nominative case. The question "Can category $\mathrm{T}$ really assign nominative case to NP?" still deserves careful thinking. The present deems that C instead of T assigns nominative case to NP. This hypothesis is supported by syntactic evidence.

The syntactic evidence comes from a very special case consideration-exceptional case marking. To examine this, take the following example:

Mary believed/considered/reported/him to love her.

In these sample sentences, the matrix verbs believe consider and report can assign accusative case to him. And these matrix verbs select infinitival TP-clause as their complement. The situations where the verbs select an infinitival TP-clause as their complement and allow phonologically overt NP with its accusative case (him) in the subject position of the infinitival clause, are defined as "exceptional case marking"(Bowers, 1993).

Now the question is what on earth prevents NP (him) from being assigned nominative case and accusative case instead when NP occupies a subject position. The present study assumes that the complement after the matrix verbs is an infinitival TP-clause not a CP-clause, so NP can not be properly assigned a nominative case. As a compromise, to fulfill the requirement that NP should have an overt phonetic content, it can only be assigned an accusative case. In this sense, as the head of infinitival TP-clause, T can not assign nominative case to NP. Then these sentences are converted into the following forms, the situation will change.

Mary believed/ considered/reported that he loves her.

In these sample sentences, since the complement of the matrix verb into finite has been converted into CP-clause, NP is assigned nominative case. This indicates $\mathrm{C}$ as the head of the $\mathrm{CP}$ is capable of assigning nominative case to NP.

From the above analysis, a conclusion can be drawn: Nominative case is assigned by $\mathrm{C}$ not by $\mathrm{T}$.

And in this sense, Chomskyan case assignment rules should be rewritten as:

a. NP is nominative if governed by $\mathrm{C}$

b. NP is objective if governed by $\mathrm{V}$ with the sub categorization feature: __ NP (i.e., transitive)

This conclusion is rather important in the following aspects: 1) It provides strong evidence suggesting rightward merge order. But different from the hypothesis mentioned just now that $\mathrm{T}$ enters derivation first, then merges NP and assigns nominative case to NP; at the beginning of the sentence, $\mathrm{C}$ merges NP and assigns NP the nominative case to 
make NP subject of the sentence. 2) It challenges UTAH. UTAH posits that NP is located at the inner specifier position of $\mathrm{T}$ in order to receive nominative case from T. Since NP is assigned nominative case by C, UTAH practically loses its syntactic significance to sentence derivation, which will be further discussed in chapter six.

The above analysis of case indicates that if a bottom-up merge order is observed, the concerning syntactic elements can not be assigned case properly.

\section{B. Evidence of Agreement}

Agreement is another important consideration for syntactic studies. According to Chomsky, two syntactic elements are said to agree in respect of some grammatical features if they have same value for the relevant features. Take a simple sentence for example He smokes., in this sentence, the verb smokes is said to agree with its subject he because both are third person singular expressions.

Concerning agreement, English syntax mainly focuses on the agreement of person and number feature while other languages also care about gender feature. Roughly examined, English sentences have three categories of agreements (Branigan, 1992):

The first category will be the agreement between subject and verb, and as a matter of fact, in G-T grammar, it is T that is in charge of checking the agreement features of person and number. The example taken above best represents this category. The second category is the agreement between subject and object. In the sentence like They are students. The object students is said to be in agreement with the subject they in terms of number. The last category is the agreement between indirect object and direct object in double object construction. The crew handed back the passengers their passports. In this example, the indirect object passengers are plural, as a consequence only the plural form their passports can be used.

In earlier G-T syntax, Chomsky made attempts to study agreement from the representational perspective. For each category of agreement, he defined three levels of representation, Agr Agr (Agree bar) and Agr P. Then corresponding to three categories, then there will be AgrS AgrS- AgrSP, AgrO AgrO- AgrOP, AgrIO AgrIO- AgrIOP(Chomsky,1991). Agr is a basic syntactic level with agreement features unvalued which has an intermediate projection into Agr-. Agr- is the level where another syntactic element is located and prompts to value the unvalued features. A maximal projection is Agr $\mathrm{P}$ which constitutes an agreement phrase.

In recent phase stage, with bar level deleted, Chomsky endowed agreement a derivational explanation within the syntactic operation of merge(Chomsky,2005). When the syntactic operation merge is carrying on, the interpretable features of the existing syntactic element will check the uninterpretible features of another syntactic element and assists to delete these uninterpretible features to establish the agreement. Take He smokes. for example, the person and number features of the subject are interpretable while the suffix indicating the third person singular "s" can not be interpreted syntactically. If the merge operation wants to go on, all the uninterpretible features should be deleted. So, when the merge of he and smokes takes place, the interpretable features of he will check and delete the uninterpretible features of suffix s, and then the agreement relation can be established.

The bottom-up merge order will cause problem for agreement between subject and T. As has been mentioned, in the process of derivation subject NP with its own interpretable features of person and number, assists to check and delete the uninterpretible features of $\mathrm{T}$ (syntactically marked by suffix). According to bottom-up merge order and UTAH, subject NP enters derivation first. There seems to arise no problem for the agreement relation between subject and T. But this will cause the contradiction between the requirement of case assignment and requirement of agreement feature valuing. This contradiction was ignored by most of the former syntactic studies. If as bottom-up merge order and UTAH demand, subject NP enters derivation first and is located at the inner specifier position of T, NP is able to value the uninterpretible agreement feature of T. However in this way, the nominative case of subject can not be assigned properly since $\mathrm{T}$ enters later. If the reverse order is followed, subject NP enters derivation but is located at the outer specifier position of T, the nominative case of subject NP can still not be properly assigned. The only solution is to have $\mathrm{C}$ assign nominative case to subject NP and NP value the uninterpretible agreement feature of T. Thus the proper merge order is $\mathrm{C}$ merges subject and then merges $\mathrm{T}$.

For the agreement between subject and object, the discussion will be much simpler. Take the sentence They are students. for example. There exists an agreement relation between they and students as far as number feature is concerned. This kind of agreement phenomenon is special since it can not be explained under the background of phase theory. And most probably, the recent phase theory seems to pay little attention to giving this phenomenon a satisfying explanation. As a matter of fact, the agreement between subject and verb (T) can be reconsidered as a kind of governing since subject and $\mathrm{T}$ are within the domain of same phase. And this kind of agreement relation is established through checking and deleting the uninterpretible features. While for the agreement relation between subject and object, since subject and object belong to different phases (subject to TP while object to VP), it can not be explained in terms of governing relation or checking and deleting uninterpretible features. But one thing quite certain is that semantically or logically speaking, first there will be plural feature of the subject then the plural feature of object. The plural feature of subject predetermines that of the object not the opposite. The match between the plural feature of the subject and that of the object, essentially speaking, reveals a kind of binding relation. (The important thing for this relation is a syntactic element located at the left of the sentence semantically or logically binds another located at the right of the sentence. For detailed analyses, see the following section about anaphor.) So, in a typical derivation of a sentence, NP students 
used as the object of the sentence can not enter derivation first as the bottom-up merge order dose, because if subject NP dose not enter the derivation first, the uninterpretible features of object NP subsequently can not be checked and deleted.

The agreement between direct and indirect objects also provides strong evidence against the bottom-up merge order. G-T grammar, influenced by case grammar, also deems that different theta-roles possess different importance in syntactic structure (Larson, 1988). In a typical double object construction, as far as the importance of different theta-roles possess is concerned, agent (subject NP) is more important than goal (indirect object NP) and than patient (direct object NP). And in terms of sentence derivation, the theta-role with less importance enters derivation first. So when the derivation of ditransitive structure is going on, according to bottom-up merge order, first there will be the merge of $\mathrm{V}$ and direct object and then indirect object merges from left to the existing constituent. Thus, in a typical tree diagram of double object structure, direct object is located at the very bottom of the tree diagram. After the merge of $\mathrm{V}$ and direct object, indirect object enters derivation.

Take The crew handed back the passengers their passports. for example, if the bottom-up merge order is followed, their passports enters derivation first. As a matter of fact, it can't enter derivation first since its plural number feature has not been valued.

As a conclusion, the careful examination of three different categories of agreement indicates bottom-up merge order can not properly establish any of them, while rightward merge order can.

\section{Evidence of Anaphor}

Among all the factors leading to doubt about the bottom-up merge order, anaphor is especially important since it provides the strongest evidence though the phenomenon itself may not occupy a remarkable position in syntactic studies, even less noticed by phase theory.

A review of the phenomenon of anaphor under the background of G-T grammar is indispensable to the present study. In government and binding theory, as far as referential properties are concerned, overt DPs fall into three different classes: anaphors, pronouns and referential expressions. The group of anaphors includes reflexives such as himself, themselves and reciprocals such as each other. The group of pronouns includes the usual pronouns such as she, him. Finally, the group of referential expressions includes proper names such as Mary and Bill, and referring DPs such as the coach and players.

Anaphors have the distinctive property that they are dependent on an antecedent included in the sentence for their reference. In other words, they must have an antecedent in the sentence in which they occur, as shown in the following pair of sample sentences:

1a. The players blame themselves/each other.

1b. *The coach blamed themselves/each other.

In the first sentence, the anaphor themselves/each other has an antecedent, namely the players. In the second sentence, however, the anaphor does not have an antecedent, and hence the fact that the sentence is excluded. The subject the coach does not qualify as antecedent for the anaphor in the second sentence because the subject does not share the same number feature with the anaphor. (The ungrammaticality of the second sentence also confirms that the subject enters derivation first and then the agreement between subject and object can be established.) Differences in the features of DP imply differences in the referential values indicated in terms of indices. The subject and the anaphor have different features in the second sentence and therefore different indices.

Different from anaphors, pronouns do not necessarily require an antecedent in the sentence although they may have one, as the following sentences illustrate.

1. The coach suspects that the players blame him.

2. The coach (i) suspects that the players blame him (i).

3. The coach (i) suspects that the players blame him (j).

Unlike pronouns and anaphors, referential expression cannot have an antecedent in the sentence, for example:

1. He suspects that the players blame John.

2. *He(i) suspects that the players blame John(i).

3. He (i) suspects that the players blame John (j).

From the above analyses of the difference of anaphors, pronouns and referential expressions, it can be understood whether antecedent is necessary will decide the grammaticality of the sentence. And Chomsky defines the notion antecedent as the category which bears the same index as an anaphor or a pronoun and further defines the conditions for antecedent. That is, the antecedent of an anaphor or a pronoun must i) bear the same person and number feature as the anaphor or pronoun ii) c-command the anaphor or pronoun

And basing on the relation between antecedent and anaphor or pronoun, Chomsky defines the concept of binding and the well-known binding conditions (Chomsky,1981).

Binding

$a$ binds $b$ if and only if

i) $a$ is co-indexed with $b$

ii) a c-commands b

Binding condition A 
An anaphor must be bound within its local domain

Binding condition $\mathrm{B}$

A pronoun must be free in its local domain

Binding condition $\mathrm{C}$

An r-expression must be free

The above is a very brief review of the syntactic study of anaphor and concerning binding phenomenon. The bottom-up merge order fails to establish this binding relation between anaphor and its antecedent. Take the following sentence for example:

The players blamed themselves/each other.

If the bottom-up merge order is followed, the first syntactic element which enters derivation must be themselves and each other, and then verb merges them to constitute a VP, which means, the computational system first chooses a reflexive themselves or a reciprocal each other from the lexicon to be identified lexical items. This will be problematic since these lexical items of themselves and each other should be bound by the antecedent the players or the phonetic content of themselves and each other should be predetermined by the antecedent the players; while at this stage of derivation, according to the bottom-up merge order, the antecedent has not entered derivation yet. So, how can lexical items of themselves and each other be identified and directly chosen from the lexicon without their antecedent entering derivation? If the merge order is changed, NP the players enters derivation first, and it predetermines the phonetic content of reflexive and reciprocal through proper binding relation. Subsequently, the lexical items of themselves and each other can be specified and chosen by the computational system and the problem will be solved.

As a matter of fact, the term antecedent implies a proper merge order since both the prefix ante and the term antecedent semantically mean before which suggests that antecedent should enter derivation first and anaphor or reciprocal later.

Semantically or logically, the binding relation relating anaphor to its antecedent demands a rightward merge order since the essence of binding relation is the syntactic element to the left of the sentence logically binds another one to the right of the sentence.

The above three sections studied how a bottom-up merge order failed to assign case, establish agreement and bind antecedent and anaphor. As a matter of fact, the considerations of case, agreement and anaphor concerned either governing or binding relation and these relations in turn are essentially logical. The proper establishment of logical relation like governing and binding requires that a rightward merge order be adopted instead of bottom-up merge order or leftward merge order. Then the following section would provide two more specific piece of syntactic evidence for rightward merge order.

\section{Specific Evidence}

VP-fronting constructions support rightward merge order and challenge bottom-up merge order. VP -fronting phenomenon can be revealed in conversion of the following sample sentence:

1. John intended to give candy to children in libraries on weekends and he did give candy to children in libraries on weekends.

This is a compound sentence, and as far as the later part of this compound sentence is concerned, for pragmatic purpose of emphasizing the information (information focus), the VP phrases can be moved to the beginning to form a VP-fronting construction(James,1993). But there are some principles to follow; otherwise the VP-fronting can be ungrammatical. For example, the following conversions are acceptable

1. John intended to give candy to children in libraries on weekends and give candy he did to children in libraries on weekends.

2. John intended to give candy to children in libraries on weekends and give candy to children he did in libraries on weekends.

3. John intended to give candy to children in libraries on weekends and give candy to children in libraries he did on weekends.

4. John intended to give candy to children in libraries on weekends and give candy to children in libraries on weekends he did.

But not

1.... and on weekends he did give candy to children in libraries.

2.... and in libraries on weekends he did give candy to children.

3. ... and to children in libraries on weekends he did give candy.

Then the acceptability of VP-fronting proves that bottom-up merge order is inappropriate.

According to the bottom-up merge order, the derivation of the later part of the compound sentence will be: 


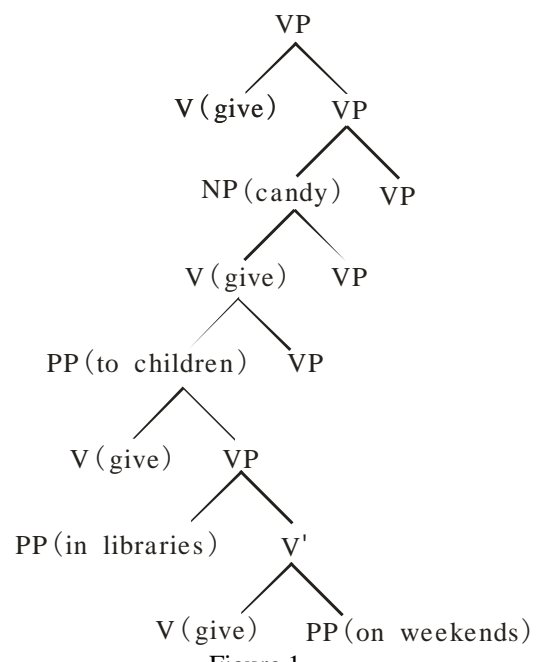

Thus, fronting give on weekends, give in libraries on weekends or give to children in libraries on weekends is plausible. (Here the verb give has been raised to the predicate position so the trace in the overt construction should be deleted, that is why in the final presentation the structures are and on weekends he did give ...not and give on weekends he did give ....)

But as a matter of fact, all the possible VP-fronting constructions listed above are practically ungrammatical. The problem arises not from VP-fronting construction itself but from the imagined bottom-up merge order.

If the second part of the compound sentence is derived in a rightward merge order, what will be the result?

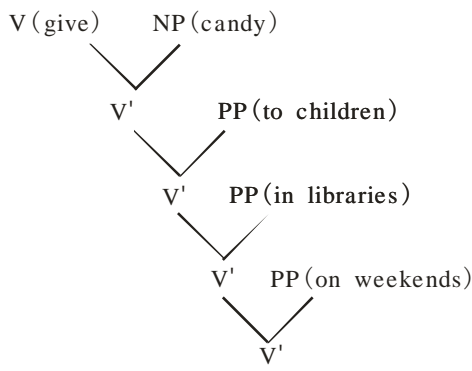

Figure 2

Thus, fronting give candy, give candy to children, give candy to children in libraries or give candy to children in libraries on weekends are plausible according to the order they enter derivation. And as a matter of fact, all these VP-fronting constructions are proved to be grammatical. This indicates the rightward merge order can satisfactorily explain the VP-fronting construction.

In English, to express the meaning of change of hand of something represented by word give, either double object (ditransitive) construction or dative construction (Barss \& Lasnik, 1993). The ditransitive construction will be give somebody something. In this construction, somebody is the indirect object while something is the direct object. The dative construction will be give something to somebody. And in this construction, something is the object while the prepositional phrase is the complement.

Compared with dative construction, ditransitive construction, among all the English syntactic constructions, is very special. In English, hardly anywhere else can a syntactic construction be found in which a verb may have two objects except in this case of indicating change of hand. (This is quite different from Chinese. In Chinese, there are four kinds of structures in which a verb can be followed by two objects (James, 2003). And in English, this change of hand means from the hand of agent to the hand of patient, not the opposite.) While dative construction though has the same meaning as that of ditransitive construction, it has nothing syntactically special. Syntactically speaking, dative construction is a verb followed by an object and then followed by a complement. In English, this is a commonly found structure not necessarily indicating change of hand. For example, We put the book on the desk. or We consider him a good student. In both of the two sample sentences, a verb is followed by an object and then a complement.

The uniqueness of the ditransitive construction may offer an insight into the merge order problem.

The present study deems that the syntactic uniqueness of English ditransitive construction has something to do with the semantic uniqueness of main verbs indicating change of hand. Take the most representative word give for example, give may have somebody be its object, in which case, somebody indicates the goal of the act of give, and this somebody has no physical contact with the person who gives him that certain thing. This is quite like some other English verbs as 
blame. In the sentence like We blame him., the accusative case him indicates the goal of the act, while he has no physical contact with the subject of the sentence we.

And an alternative is give can also choose something as its object, in which case, something is the patient of the act of give, which means something will have the physical contact with the person who gives that thing. In other words, something is the object that give directly acts on.

So the syntactic uniqueness of the ditransitive construction arises from the uniqueness of that particular category of verbs, which can choose either something or somebody as their objects. The unique semantic features of this type of verbs predetermine the features of that syntactic construction.

Theoretically, the different constructions of ditranstive and dative structures are determined by the choice the main verb makes to allow either somebody or something enters derivation first. If the verb chooses somebody as its object then constitutes the construction, give somebody something. If the verb chooses something as its object then constitutes construction, give something somebody. This construction is ungrammatical. When give choose something as its object first, something establishes a logical semantic relation with somebody; And since somebody is the goal of something aims at. Therefore, a preposition to indicating this relation should be added.

As a conclusion, the unique semantic feature of verb indicating the change of hand predetermines the later difference of different syntactic constructions. After main verb enters derivation, if the computational system choose a NP indicating person, the derivation will finish with a ditransitive otherwise it will be a dative.

However, if the bottom-up merge order is followed, for ditransitive structure, $\mathrm{V}$ merges something first and both of them are located at the bottom of the tree diagram of that structure and for dative, V merges somebody first and they are located at the bottom. This is evidently against the above analysis done concerning the lexical choice which leads to the difference between structures since for ditransitive, lexically, V semantically first chooses somebody as its object while for dative, lexically, V semantically first chooses something as its object. The only solution to the problem is change merge order. If a rightward merge order is followed, for ditransitive structure, syntactically $\mathrm{V}$ merges something first and this matches that lexically $\mathrm{V}$ chooses something first. And for dative structure, it will be the same case.

\section{RightWARD MERGE ORDER}

The above analysis of the bottom-up merge order, or linearly, a leftward merge order suggests that it is contradictory to the logical truth and therefore syntactically unacceptable. This unacceptability essentially attributes to the theoretical foundation for arguing bottom-up merge order, namely the verb-centered constituent system and the constituent-centered computational system. Before a new merge order is advocated, it is necessary to reassess the defects of this theoretical foundation. And based on this reassessing work, a new merge order can be established.

The verb-centered constituent system requires that verb (in the more recent stage replaced by T) and NP first merge and then project a VP or TP. Otherwise if subject and C merge first it can not project a VP and fail to establish a predication relation as has been illuminated. For this consideration, the whole derivation has to start from the very right. However, since the computational system is derivational, it can be flexible. That is to say, after $\mathrm{C}$ and subject merge to project a $\mathrm{CP}$, subject can still be used to merge $\mathrm{T}$ to project a TP and meanwhile establishes proper predication relation.

Besides, the constituent-centered computational system requires that all the syntactic computation should be implemented in a constituent system. That is to say, as the basic unit of syntactic operation, a syntactic element enters derivation first and then a new syntactic element merges from left to the existing element and project a constituent. Within the domain of the constituent, the newly merged syntactic element govern the existing ones and is in charge of deleting the concerning uninterpretible syntactic features. After this stage, a new syntactic element enters derivation from left again and this element and the existing constituent project a new constituent. Thus this newly merged syntactic element constitutes a c-commanding relation with the syntactic elements within the domain of the first constituent. And this element also establishes a binding relation with the original ones if there is a possible one. But Chomsky seems to forget the governing and binding relations (For many scholars, Chomsky did his best work in illustrating governing and binding relation and therefore it is the highlight of his theory.) essentially reflect a logical relation. The proper logical relation should be a relation between the existing element controls and the newly merged element. On the contrary, the bottom-up merge order as a matter of fact establishes a relation that the newly merged element controls the existing element. In this sense, the bottom-up merge order violates the logical truth.

The following are the specific procedures of rightward merge order.

Rightward merge order

1. C element enters derivation first and then merges subject. As a functional category without specific lexical meaning, $\mathrm{C}$ is undoubtedly important and therefore can not be ignored. One of the important arguments of this dissertation is the functional category $\mathrm{C}$ is in charge of assigning case to subject, not the traditional assumption that nominative case is assigned by $\mathrm{T}$. Thus, $\mathrm{C}$ establishes a governing relation with subject. When subject enters derivation, its case feature is uninterpretible and needs to be checked. At this time, the existing $\mathrm{C}$ category assigns case to subject and consequently deletes the uninterpretible case feature of subject and both $\mathrm{C}$ and subject can be spelled out to possess phonetic content. This is the first stage of the derivation.

2. The second stage of the derivation will be the merge of a new functional category $\mathrm{T}$ from right to the existing $(\mathrm{C}$ subject). It is reasonable to keep this functional category in derivation since the syntactic meaning indicated by 
functional category $\mathrm{T}$ like tense, aspect, voice and mood is universal. And for $\mathrm{T}$, its agreement features of person and number are uninterpretible and need to be checked. And subject, as a syntactic element enters derivation earlier, can use the agreement features of itself to check and delete the uninterpretible features of $T$. Thus a governing relation has been established between subject and T. And also during this stage of derivation, a predication relation is established and thus an external argument structure is built up. When this finishes, $\mathrm{T}$ can be spelled out and thus we have (C subject T).

3. The third stage of the derivation will be the merge of $\mathrm{v}$ or $\mathrm{V}$ to the existing ( $\mathrm{C}$ subject $\mathrm{T})$. An alternative or is chosen for the reason that different from Chomskyan phase theory, the present study deems that $\mathrm{v}$ is not a necessary syntactic element for all sentences. The functional category v classified by Chomsky is not applicable to any sentence structure. This will be left for detailed analyses in the chapter illuminating the successive cyclical move pattern. But the main argument is the original purpose for Chomakyan introducing this functional category is to solve the syntactic derivation of verbs with three-place predicate structure (or three-place verb structure). Supposing in three-place predicate structure like double object sentence, there is only $\mathrm{V}$ element which helps to locate object and assign accusative case to one object, the remaining object can not be properly located and assigned a case. As a result, a functional category $\mathrm{v}$ is specially designed for these three place predicate structures. Since $\mathrm{v}$ or $\mathrm{V}$ can be two alternatives for the third stage of derivation, it will be discussed separately.

If the sentence construction dose need a functional category $\mathrm{v}$, then the third stage will be that $\mathrm{v}$ enters derivation and merges from right to ( $\mathrm{C}$ subject $\mathrm{T}$ ). During this stage of derivation, there is no uninterpretible feature for both $\mathrm{T}$ and $\mathrm{v}$. A syntactic interpretation for this would be these two elements are syntactically equal. But in languages like English, $\mathrm{T}$ in many cases (except for negative or interrogative sentences or for pragmatic emphasis) can attract $\mathrm{v}$ to its position while $\mathrm{T}$ can reform itself to be a suffix. Our interpretation for this is since $\mathrm{T}$ can attract $\mathrm{v}$ from the original position to the position of its own not the vice versa, it indicates they are syntactically unequal at least in languages like English. Basing on this, we suppose $\mathrm{T}$ establishes a governing relation with $\mathrm{v}$. And v is spelled out then we have (C subject $\mathrm{T}$ v)

If $\mathrm{v}$ is not necessary for sentence construction, then $\mathrm{V}$ enters derivation. And $\mathrm{T}$ establishes a governing relation with $\mathrm{V}$. $\mathrm{V}$ is spelled out then we have $(\mathrm{C}$ subject $\mathrm{T} \mathrm{V})$.

4. The next stage has two possibilities as well. One is indirect object (for double object) merges form right to (C subject $\mathrm{T} \mathrm{v}$ ). The alternative might be an object (for object complement structure) merging from right to(C subject $\mathrm{T}$ v). $\mathrm{v}$ establishes a governing relation with indirect object or object and assigns case to this indirect object or object, and therefore the internal argument are constructed at the same time. Then there will be $(\mathrm{C}$ subject $\mathrm{T} v$ indirect object) or $(\mathrm{C}$ subject $\mathrm{T}$ v object)

The other one is simpler, object merges from right to $(\mathrm{C}$ subject $\mathrm{T} \mathrm{V})$. V establishes a governing relation with this object and assigns case to it, constructing an internal argument structure. Thus there will be (C subject T V object). For sentences with one place or two place verb structure, the whole derivation ends here.

5. For three place predicate structure, there is still another and final stage. Same as the traditional derivation, $\mathrm{V}$ element is introduced to locate the remaining syntactic element. Direct object or complement merges from right to (C subject $\mathrm{T} v$ indirect object) or $(\mathrm{C}$ subject $\mathrm{T} \mathrm{v}$ object) to constitute ( $\mathrm{C}$ subject $\mathrm{T} \mathrm{v}$ indirect object $\mathrm{V}$ direct object) or $(\mathrm{C}$ subject $\mathrm{T}$ v object $\mathrm{V}$ complement).

And during this stage, IO and DO, or object and complement are syntactic elements with specific semantic reference. They establish a binding relation to check whether they agree in number feature like sentences We give them their passports back. and We consider them good students. illustrate.

From the above analyses, it can be clearly seen that in each stage of derivation following the rightward merge order, an existing syntactic element establishes a governing relation with the newly merged syntactic element. The existing syntactic element's governing relation to the newly merged syntactic element ensures that the syntactic operations like case assigning, agreement feature checking, verb-attracting can be properly implemented and the uninterpretible grammatical features of the syntactic elements can be successfully valued. Thus the derivation following rightward merge order, on the one hand, successfully constructs a sentence and on the other hand, values almost all uninterpretible grammatical features through governing relation except for those edge feature which can only be valued through move operation.

\section{REFERENCES}

[1] Andrew, R. (2000).Syntax: a Minimalist Introduction. Beijing: Foreign Language Teaching and Research Press.

[2] Andrew, R. (2008).Minimalist Syntax: Exploring the Structure of English. Beijing: Foreign Language Teaching and Research Press.

[3] Andrew, R. (2004).Syntactic Theory and the Structure of English a Minimalist Approach. Beijing: Beijing University Press.

[4] Barss, L. \& Lasnik, N. (1997). A Note on Anaphor and Double Objects. Linguistic Inquiry (28):347-354.

[5] Bowers, J. (1993). The syntax of predication. Linguistic Inquiry (24): 591-656.

[6] Branigan, P. (1992).Subjects and Complementisers. Cambridge, MA: the MIT Press.

[7] Chomsky, N. (1981). Lectures on Government and Binding. Dordrecht: Foris.

[8] Chomsky, N. (1991). Some Notes on Economy of Derivation and Representation. In his Principles and Parameters in Comparative Grammar. Cambridge, MA: the MIT Press.

[9] Chomsky, N. (2005).On Phases. In Otero, C, P. Foundational Issues in Linguistic Theory. Cambridge, MA: MIT Press.

[10] Chomsky, N. ( 2001).Derivation by Phase. In Hale, K. A Life in Language. Cambridge, MA: the MIT Press. 
[11] James, H. (1993).Reconstruction and the Structure of VP: Some Theoretical Consequences. Linguistic Inquiry, (24). 103-138.

[12] James, H. (2003).The Syntax of Chinese. Cambridge: Cambridge University Press.

[13] Larson, R. (1988). On the Double Object Construction. Linguistic Inquiry, (19): 335-391.

[14] Manchun, Dai.(2001). Leftward Merge in Broad Sense. Beijing Foreign Languages University.

Zhiyi Zhang, Ph.D. in linguistics, earned in Nanjing Normal University, Nanjing, Jiangsu Province, China, in 2012. The major field is English and Chinese formal syntax.

$\mathrm{He}$ is now the associate professor in NJNU and is doing his post-doctoral research in Nanjing University. His main published articles include The semantic and syntactic study on light verbs, A New Interpretation to Definiteness Effect and Derivation of Existential Sentences, the Cog-Semantic Study on the Difference between Datives and Distransitives.

Dr. Zhang is now the secretary to the Jiangsu Foreign Languages Association. 